H. JORIS AND E. PRE.SSMANN

KODAI MATH. J.

13 (1990), 241-264

\title{
QUOTIENTS OF SMOOTH FUNCTIONS
}

\author{
By Henri Joris and Emmanuel Preissmann
}

\section{Introduction.}

The following theorem was proved in $[\mathrm{J}]:\left(^{*}\right)$ if $f: \boldsymbol{R} \rightarrow \boldsymbol{R}$ is such that $f^{2}$ and $f^{3}$ are of class $C^{\infty}$, then so is $f$. The proof used elementary, but rather complicated equations relating the derivatives of $f$ and of its powers. We thought it possible to imagine another proof based on the fact that $f$ is the quotient of two smooth functions $f^{3}$ and $f^{2}$, or rather that the product of $f$ by the smooth function $f^{2}$ is itself smooth. Of course a function $g$ as well as the product $f g$ may be smooth even if $f$ is not, so we had to look for additional conditions which are sufficient to imply the smoothness of $f$.

Here is one possible answer to that problem: if $f, g: \boldsymbol{R} \rightarrow \boldsymbol{C}, m \in \boldsymbol{N}$ and $\boldsymbol{\alpha}>0$ are such that $g, f g$ and $f^{m}$ are smooth and $|f| \leqq|g|^{\alpha}, f$ is smooth (Theorem 1). (*) follows immediately from this theorem if one sets $g=f^{2}, m=2, \alpha=1 / 2$.

Remark. An elegant and simple proof of $\left(^{*}\right)$, based on ring theory, has recently been given in [AM].

Theorem 1 will be used to study a family of smooth maps called pseudoimmersions (cf. [JP1]), and of which the curve $t \mapsto\left(t^{2}, t^{3}\right)$ appearing in $\left(^{*}\right)$ is but a simple example:

A $C$ - ${ }^{\infty}$ application $h: N \rightarrow M, M$ and $N$ being $C^{\infty}$-manifolds, is a pseudo-immersion if for each continuous application $f$ of a $C^{\infty}$-manifold $P$ to $N, h \circ f \in C^{\infty}$ implies $f \in C^{\infty}$.

By the condition that $f$ is continuous, each immersion is a pseudo-immersion. (If in the above definition $C^{\infty}$ is replaced by $C^{r}$, for some $r \in N$, then immersions and pseudo-immersions become the same thing.) The same condition implies that the pseudo-immersivity of a smooth map is a local property. Hence, it's enough to study maps $\boldsymbol{R}^{n} \rightarrow \boldsymbol{R}^{m}$, or even germs of smooth maps $\left(\boldsymbol{R}^{n}, 0\right) \rightarrow$ $\left(\boldsymbol{R}^{m}, 0\right)$. As it was proved in [J], the pseudo-immersivity follows if the condition of the definition is checked for $P=\boldsymbol{R}$; thus, by $\left(^{*}\right)$, the non-immersive map $t \mapsto\left(t^{2}, t^{3}\right)$ is a pseudo-immersion.

In [JP1] the pseudo-immersions $N \rightarrow M$ for $\operatorname{dim} N=1$, were completely described (by determining the pseudo-immersive germs $(\boldsymbol{R}, 0) \rightarrow\left(\boldsymbol{R}^{m}, 0\right)$ ). For $\operatorname{dim} N \geqq 2$, the task appears to be much more difficult, except in the case where $\operatorname{dim} M=$

Received December 22, 1989. 
$\operatorname{dim} N$ (then any pseudo-immersion is an immersion, that is, a local diffeomorphism) or in the case where $\operatorname{dim} N>\operatorname{dim} M$ (then there are no pseudo-immersions) (cf. [JP2]). However, Theorem 1 enables us to find some new families of pseudoimmersions (Theorems $2^{\prime}$ et 3 ).

In part 4 we give some examples and counterexamples, disproving or confirming a few guesses inspired by the study of the cases $\operatorname{dim} N=1$ and $\operatorname{dim} N=$ $\operatorname{dim} M$.

In the hypothesis of Theorem 1, all four conditions are necessary: if any of them is omitted the conclusion is no more valid. This is quite obvious except for the condition $f^{m} \in C^{\infty}$; if it is omitted, a counterexample is given in the fifh part, where the following is proved: If $f, g: \boldsymbol{R} \rightarrow \boldsymbol{C}$ and $\alpha>0$ are such that $g$ and $f g$ are smooth, and $|f| \leqq|g|^{\alpha}$, then $f \in C^{[\alpha]}$; if moreover $f$ is real, then $f \in$ $C^{[2 \alpha]}$. These conclusions are best possible.

Acknowledgements: In the third part, where we use some results of algebraic geometry, Prof. M. Ojanguren's suggestions were very precious for us. We are indebted to the Fonds national suisse de la recherche scientifique for financial support.

Notations: 0 is not a natural number, so $N=\{1,2,3, \cdots\}$. If $I$ is a real interval, its length is denoted by $|I|$. A smooth mapping is a $C^{\infty}$-mapping. A mapping $f: \boldsymbol{R}^{n} \rightarrow \boldsymbol{C}^{m}$ is called flat at $x_{0}$ if for any $s \in \boldsymbol{N}$ one has $\left\|f(x)-f\left(x_{0}\right)\right\| \ll$ $\left\|x-x_{0}\right\|^{s}$ as $x \rightarrow x_{0}$; if $f$ is smooth, this implies that all the derivatives of $f$ at $x_{0}$ vanish. By g.c.d. we refer to the greatest common divisor and by [ ] to the integral part. Q.E.D. denotes the end of the proof.

\section{Smoothness of quotients of smooth functions.}

Our main concern in this section is with the proof of the following theorem.

Theorem 1. Let $f, g: \boldsymbol{R} \rightarrow \boldsymbol{C}$ be two functions, $m$ a natural integer, and let $C, \alpha$ be two positive constants such that

a) $g, f g, f^{m} \in C^{\infty}(\boldsymbol{R}, \boldsymbol{C})$;

b) $|f(x)| \leqq C|g(x)|^{\alpha}$ for every real $x$.

Then $f \in C^{\infty}(\boldsymbol{R}, \boldsymbol{C})$.

In section 3 we shall apply this theorem to prove the pseudo-immersivity of certain families of germs. Here we deduce just one easy consequence.

CoRollary. (see $[\mathrm{J}]$ for $f$ real; [DKP], [JP1] [AM] for $f$ complex) If $f: \boldsymbol{R} \rightarrow \boldsymbol{C}$ and $r, s \in \boldsymbol{N}$ are such that g.c.d. $(r, s)=1, f^{r} \in C^{\infty}, f^{s} \in C^{\infty}$, then $f \in C^{\infty}$.

Proof of the corollary. It is easy to show, [J], that $f^{r s}$ and $f^{r s+1}$ are smooth. 
The conclusion then follows by Theorem 1 with $m=r s, g=f^{r s}, C=1, \alpha=1 / r s$.

Q.E.D.

We begin the proof of Theorem 1 by three simple lemmas:

LEMMA 1. Let $f, g: \boldsymbol{R} \rightarrow \boldsymbol{C}$ be functions. Suppose that $g$ and $f g$ are smooth, that $g$ is not flat at $b$ and that $f$ is bounded near $b$. Then $f$ is smooth near $b$.

Proof. By Taylor's theorem and by the hypothesis we may find a natural number $n$, such that $g(x)=(x-b)^{n} \gamma(x), \gamma$ smooth, $\gamma(b) \neq 0$, and $(f g)(x)=(x-b)^{n} \varphi(x), \varphi$ smooth; hence $f=\varphi / \gamma$ is smooth near $b$.

Q.E.D.

LEMMA 2. If $f \in C^{n-1}(\boldsymbol{R}, \boldsymbol{C})$ is flat at $b$, and of $f^{(n)}(b)$ is defined, then $f(b)$ $=f^{\prime}(b)=\cdots=f_{(b)}^{n}=0$.

Proof. The conclusion follows from Peano's rarely used version of Taylor's Theorem (cf. [F], p. 228):

$$
f(x)=\sum_{k=0}^{n} \frac{f^{(k)}(b)}{k !}(x-b)^{k}+o\left(|x-b|^{n}\right) . \quad \text { Q.E.D. }
$$

LeMma 3. For each $n \in \boldsymbol{N}$ there is a constant $K_{n}>0$ such that

$$
\sup _{\alpha \leqq x \leqq b}|f(x)| \geqq K_{n}(b-a)^{n} \inf _{a \leqq x \leqq b}\left|f^{(n)}(x)\right|
$$

for all $f \in C^{n}([a, b], \boldsymbol{R})$.

Proof. We proceed by induction. Without loss of generality we may suppose that $a=-b, f^{(n)} \geqq 1$ in $[-b, b]$, and $f^{(n-1)}(0) \geqq 0$. Then $f^{(n-1)} \geqq b / 2$ in $[b / 2, b]$. By the hypothesis of induction one has

$$
\sup _{[-b, b]}|f| \geqq \sup _{[b / 2, b]}|f| \geqq K_{n-1}\left(\frac{b}{2}\right)^{n-1} \frac{b}{2}=4^{-n} K_{n-1}(b-a)^{n},
$$

and the lemma is proved, because $K_{0}=1$ is obvious.

Q.E.D.

Remark. It is possible to prove that the best constant is $K_{n}=\left(n ! 2^{2 n-1}\right)^{-1}$.

LEMMA 4. Let $f: \boldsymbol{R} \rightarrow \boldsymbol{C}$ be a continuous function such that $f^{m}$ is smooth for some $m \in \boldsymbol{N}$. Let $I \in \boldsymbol{R}$ be a bounded interval. Then for each $\boldsymbol{\omega}>0$ there is a constant $k_{\omega}$ such that

$$
|f(x)| \leqq k_{\omega}|x-p|^{\omega}
$$

for any $x \in I$ and any $p$ such that $f$ is flat at $p$. (Uniform flatness.)

Proof. The points of flatness of $f$ and of $f^{m}$ are the same, therefore it suffices to prove the lemma for $f^{m}$. We may suppose that $|x-p| \leqq 1$ and $\boldsymbol{\omega} \in \boldsymbol{N}$. Set $h=f^{m}$; if $h$ is flat at $p$, then $h(p)=h^{\prime}(p)=\cdots=h^{(\omega-1)}(p)=0$. Then Taylor's 
formula gives for $x \in I, x \geqq p$ :

$$
\begin{aligned}
|h(x)| & =\frac{1}{(\omega-1) !}\left|\int_{p}^{x}(x-t)^{\omega-1} h^{(\omega)}(t) d t\right| \\
& \leqq \frac{1}{(\omega-1) !} \sup _{I}\left|h^{(\omega)}\right| \int_{p}^{x}(x-t)^{\omega-1} d t, \\
& =\frac{\sup _{I}\left|h^{(\omega)}\right|}{\omega !}(x-p)^{\omega} .
\end{aligned}
$$

For $x \leqq p$ the proof is essentially the same.

Q.E.D.

For the following lemmas we introduce the spaces

$$
F=C^{\infty}([0,1], C), \quad E=C \llbracket X \rrbracket,
$$

$X$ being an indeterminate. We endow $F$ with the topology of the uniform convergence for each derivative. For polynomials of bounded (say bounded by $N$ ) degree this topology is the same as the topology given by the norm $\|P\|=$ $\sup _{0 \leqq x \leqq 1}|P(x)|$. For the coefficients $a_{0}, \cdots, a_{N}$ of $P$ are obtained linearly from the values $P(j / N), j=0, \cdots, N$, by means of the inverse of Vandermonde's matrix $\left((j / N)^{k}\right)_{j, k=0, \cdots, N}$; on the other hand $P^{(n)}=0$, if $n>N$, and $\left|P^{(k)}(x)\right| \leqq(N+1)$ ! $\max _{i}\left|a_{i}\right|$ if $k \leqq N, 0 \leqq x \leqq 1$. (We could also have used the following general theorem: two topological vector spaces which are Hausdorff and have the same finite dimension are topologically isomorphic (see [W], p. 5-6, cor. 1 of th. 1).)

The topology in $E$ is the topology of the convergence of each coefficient: $\alpha_{n}=\sum a_{n, j} X^{j}$ tends to $\alpha=\sum a_{\jmath} X^{j}$, if and only if $a_{n, \jmath} \rightarrow a_{\jmath}, j=0,1,2, \cdots$. Generally, if $\alpha=\Sigma a, X^{j}$, it is not possible to replace $X$ by a complex variable. However, we may define $\alpha(0)=a_{0}$, and so $\alpha^{(k)}(0)=k ! a_{k}$, by formal derivation.

As well in $F$ as in $E$, we write $\alpha \rightarrow 0$ for a sequence which converges to 0 , but the same expression also means that $\alpha$ belongs to arbitrary small neighbourhoods of 0 (0-filter). We denote such a sequence (or filter) by $o(1)$.

For any $f \in F$ we denote its Taylor series at 0 by $T f$. The mapping $T: F$ $\rightarrow E$ is continuous, and $f^{(k)}(0)=(T f)^{(k)}(0)$. A polynomial $f$ will be identified with its Taylor series: $f=T f$.

If $M \in N$ or $M=0$, an identity (equality, limit, etc.) $\left(\bmod X^{M}\right)$ will mean that we take into account only the coefficients of $X^{0}, X^{1}, \cdots, X^{M-1}$. If we derive a relation $\left(\bmod X^{M}\right)$ we get a relation $\left(\bmod X^{M-1}\right)$.

LEMMA 5.

a) Let $u \in C^{\infty}(\boldsymbol{R}, \boldsymbol{C})$ be flat at $0, n \in \boldsymbol{N} \cup\{0\}, i>0$; and let $I_{k}=\left[a_{k}, b_{k}\right], k=$ $1,2,3, \cdots$, be a sequence of intervals, $a_{k}<b_{k}, a_{k} \rightarrow 0, b_{k} \rightarrow 0$, such that

$$
\sup _{I_{k}}\left|u^{(n)}\right| \gg\left|I_{k}\right|^{2} \quad \text { for } \quad k \rightarrow \infty \text {. }
$$

Then there is an integer $m, n \leqq m<n+i$, such that (for an appropriate subsequence) 


$$
\inf _{I_{k}}\left|u^{(m)}\right| \gg\left|I_{k}\right|^{2+n-m},
$$

and for any $l>m$

$$
\sup _{I_{k}}\left|u^{(l)}\right|=o\left(\left|I_{k}\right|^{2+n-l}\right) .
$$

Also there is a sequence of polynomials $U_{k}$, deg $U_{k}<i+n,\left\|U_{k}\right\| \gg 1$, such that one has (in $F$ ):

$$
\left|I_{k}\right|^{-\imath-n} u\left(b_{k}-\left|I_{k}\right| x\right)=U_{k}(x)+o(1) .
$$

b) If the sequence $I_{k}$ is as above, if $u \in C^{\infty}(\boldsymbol{R}, \boldsymbol{C})$ is flat at 0 , and if $s>0$, then there is a sequence of polynomials $U_{k}$, $\operatorname{deg} U_{k}<s$, such that we have in $F$

$$
\left|I_{k}\right|^{-s} u\left(b_{k}-\left|I_{k}\right| x\right)=U_{k}(x)+o(1) \text {. }
$$

Proof. a) We choose $m$ to be the greatest integer having the property

$$
\sup _{I_{k}}\left|u^{(m)}\right| \neq o\left(\left|I_{k}\right|^{2+n-m}\right) .
$$

Then (3) is an immediate consequence. By (1) and the flatness of $u$ at 0 we have $m \geqq n$ and $m<n+i$. Replacing the sequence $I_{k}$ by a suitable subsequence we obtain

$$
\sup _{I_{k}}\left|u^{(m)}\right| \gg\left|I_{k}\right|^{2+n-m}
$$

Therefore (using (3)),

$$
\begin{aligned}
\sup _{I_{k}}\left|u^{(m)}\right|-\inf _{I_{k}}\left|u^{(m)}\right| & \leqq \int_{a_{k}}^{b_{k}}\left|u^{(m+1)}(y)\right| d y \leqq\left(b_{k}-a_{k}\right) \sup _{I_{k}}\left|u^{(m+1)}\right| \\
& =o\left(\left|I_{k}\right|\left|I_{k}\right|^{i+n-m-1}\right)=o\left(\left|I_{k}\right|^{2+n-m}\right),
\end{aligned}
$$

and (2) is implied by this and by (4). Set

$$
V_{k}(x)=\left|I_{k}\right|^{-\imath-n} u\left(b_{k}-\left|I_{k}\right| x\right) .
$$

We then have

$$
V_{k}^{(l)}(x)= \pm\left|I_{k}\right|^{-\imath-n+l} u^{(l)}\left(b_{k}-\left|I_{k}\right| x\right) .
$$

By (3), we find that $V_{k}^{(m+1)} \rightarrow 0$ in $F$. If we set $U_{k}(x)=T_{m} V_{k}(x)$ (which is the $m^{t h}$ Taylor polynomial of $V_{k}$ at 0 ) then by Taylor's formula

$$
\begin{gathered}
V_{k}(x)-U_{k}(x)=\frac{1}{m !} \int_{0}^{x}(x-t)^{m} V_{k}^{(m+1)}(t) d t \\
\left(V_{k}-U_{k}\right)^{(r)}(x)= \begin{cases}\frac{1}{(m-r) !} \int_{0}^{x}(x-t)^{m-r} V_{k}^{(m+1)}(t) d t & \text { if } r \leqq m, \\
V_{k}^{(r)}(x) & \text { if } r>m .\end{cases}
\end{gathered}
$$

But we know that $V_{k}^{(m+1)} \rightarrow 0$ in $F$, thus $V_{k}-U_{k} \rightarrow 0$ in $F$, and eventually, by (4): 


$$
\sup _{0 \leqq x \leqq 1}\left|U_{k}^{(m)}\right| \geqq \sup _{0 \leqq x \leqq 1}\left|V_{k}^{(m)}\right|+o(1) \gg 1
$$

Therefore $\left\|U_{k}\right\| \gg 1$, and the proof of a) is complete. The proof of b) is similar and even easier, so we omit it.

Q.E.D.

Lemma 6. Let $F, G$ be complex fixed polynomials, $G \neq 0$, and let $m \in \boldsymbol{N}$. Suppose that

$$
\begin{aligned}
& (G+\gamma)(F+\varphi)=G F+o(1), \\
& (F+\varphi)^{m}=F^{m}+o(1),
\end{aligned}
$$

hold in $E=C \llbracket X \rrbracket$ with $\gamma \rightarrow 0$. Then $\varphi \rightarrow 0$.

Proof. We may write $G(X)=X^{s} \Gamma(X), \Gamma(0) \neq 0, s \in \boldsymbol{N} \cup\{0\}$. Then $\Gamma$ is a unit in $E$, and $o(1) \Gamma^{-1}=o(1)$, so we may suppose $G(x)=X^{s}$. By induction on $s$ we shall prove the following sharpening of Lemma 6 :

$\left(\mathrm{A}_{s}\right)$ If $M \in N, N=M m^{s}$, and if the assumptions of Lemma 6 are verified $\left(\bmod X^{N}\right)$, with $G=X^{s}$, then its conclusion holds $\left(\bmod X^{M}\right)$.

By $(1+o(1))^{-1}=1+o(1)\left(\bmod X^{M}\right)$, we see that $\left(\mathrm{A}_{0}\right)$ is true. To prove $\left(\mathrm{A}_{1}\right)$ we suppose

$$
\begin{array}{ll}
(X+\gamma)(F+\varphi)=X F+o(1) & \left(\bmod X^{M m}\right), \\
(F+\varphi)^{m}=F^{m}+o(1) & \left(\bmod X^{M m}\right),
\end{array}
$$

and $\gamma \rightarrow 0$. We have to show that $\varphi \rightarrow 0\left(\bmod X^{M}\right)$. We shall see that if $\varphi \rightarrow 0$ $\left(\bmod X^{L}\right), 0 \leqq L<M$, then $\varphi \rightarrow 0\left(\bmod X^{L+1}\right)$; this will complete the proof of $\left(\mathrm{A}_{1}\right)$, since everything is trivial $\left(\bmod X^{0}\right)$. So suppose that

$$
\varphi=o(1)+X^{L} v \quad\left(\bmod X^{M m}\right) ;
$$

we shall prove that $v(0) \rightarrow 0$.

By (5) and (7) we find

$$
(X+\gamma) v X^{L} \longrightarrow 0 \quad\left(\bmod X^{M m}\right),
$$

and therefore

$$
(X+\gamma) v \longrightarrow 0 \quad\left(\bmod X^{M m-L}\right) .
$$

We set $X+\gamma=w$. Differentiating (8) we obtain

$$
w^{\prime} v+w v^{\prime} \longrightarrow 0 \quad\left(\bmod X^{M-L-1}\right) .
$$

From (6) and (7) it follows that

$$
w^{m-1}\left(F+o(1)+v X^{L}\right)^{m}=(X+o(1))^{m-1}\left(F^{m}+o(1)\right) \quad\left(\bmod X^{M m}\right) .
$$

Expanding products and powers and using (8), we obtain 


$$
\begin{array}{ll}
w^{m-1} X^{L m} v^{m} \longrightarrow 0 & \left(\bmod X^{M m}\right), \\
w^{m-1} v^{m} \longrightarrow 0 & \left(\bmod X^{M m-L m}\right),
\end{array}
$$

and, differentiating (10),

$$
w^{m-2} v^{m-1}\left(m\left(w^{\prime} v+w v^{\prime}\right)-w^{\prime} v\right) \longrightarrow 0 \quad\left(\bmod X^{M m-L m-1}\right) .
$$

By (9) and because $w^{\prime}=1+\gamma^{\prime}=1+o(1)$, we obtain

$$
w^{m-2}\left(v^{m}+v^{m} o(1)+v^{m-1} o(1)\right) \longrightarrow 0 \quad\left(\bmod X^{M m-L m-1}\right) .
$$

Similarly, it can be shown that

$$
\frac{d}{d X}\left(w^{a} v^{b} o(1)\left(\bmod X^{T+1}\right)\right)=w^{a-1}\left(v^{b} o(1)+v^{b-1} o(1)\right) \quad\left(\bmod X^{T}\right),
$$

if $a, b \in N$. Differentiating (11) and using (12), we obtain

$$
w^{m-3}\left(v^{m}+v^{m} o(1)+v^{m-1} o(1)+v^{m-2} o(1)\right) \longrightarrow 0 \quad\left(\bmod X^{M m-L m-2}\right),
$$

and so on; after $(m-1)$ differentiations we eventually get

$$
v^{m}+v^{m} o(1)+v^{m-1} o(1)+\cdots+v o(1) \longrightarrow 0 \quad\left(\bmod X^{M m-L m-m+1}\right) .
$$

But this implies $v(0) \rightarrow 0$, because $M m-L m-m+1=(M-L-1) m+1 \geqq 1$. This ends the proof of $\left(A_{1}\right)$.

Now suppose that $\left(\mathrm{A}_{0}\right),\left(\mathrm{A}_{1}\right), \cdots,\left(\mathrm{A}_{s-1}\right)$ hold, with $s \geqq 2$. We are going to prove $\left(\mathrm{A}_{s}\right)$. The lemma is obvious if $m=1$, so we may suppose $m \geqq 2$ which implies $N=M m^{s} \geqq 2^{s}>s$. Let $p=\sum_{0}^{N-1} a, X^{j}$ be the polynomial which is defined in a unique way by the conditions $\operatorname{deg} p<N$ and $\gamma=p\left(\bmod X^{N}\right)$. Set

$$
A=\sum_{0}^{N-1}\left|a_{j}\right| \text {. }
$$

By the hypothesis we have $p \rightarrow 0$, and therefore $A \rightarrow 0$; thus we may suppose $0 \leqq A<1$. Let $z$ be a complex variable. Then $|p(z)| \leqq A$ if $|z| \leqq 1$, and $|p(z)| \leqq$ $A|z|^{N}$ if $|z| \geqq 1$. It follows that

$$
|z|^{s}>|p(z)| \quad \text { if } \quad A^{1 / s}<|z|<A^{1 /(s-N)} .
$$

We deduce, by Rouché's theorem (cf. [N], p. 106), that $z^{s}+p(z)$ has $s$ roots $r_{1}, r_{2}, \cdots, r_{s}$ with $\left|r_{j}\right| \leqq A^{1 / s}$, while the remaining roots $\rho_{1}, \cdots, \rho_{\sigma}$ satisfy $\left|\rho_{j}\right| \geqq$ $A^{1 /(s-N)}$; here $0 \leqq \sigma \leqq N-1-s$. Hence $r_{1}, \cdots, r_{s}, \rho_{1}^{-1}, \cdots, \rho_{\sigma}^{-1} \rightarrow 0$, so

$$
\begin{aligned}
X^{s}+p(X) & =c \cdot\left(X-r_{1}\right) \cdots\left(X-r_{s}\right)\left(1-\frac{X}{\rho_{1}}\right) \cdots\left(1-\frac{X}{\rho_{\sigma}}\right) \\
& =c \cdot\left(X-r_{1}\right)\left(X^{s-1}+o(1)\right)(1+o(1)) \\
& =(X+\delta)\left(X^{s-1}+o(1)\right),
\end{aligned}
$$


where $\delta \rightarrow 0$. By the hypothesis of $\left(\mathrm{A}_{s}\right)$, we have

$$
\begin{aligned}
\left(X^{s-1}+o(1)\right)(X+\delta)(F+\varphi) & =X^{s} F+o(1) \\
& =X^{s-1} X F+o(1) \quad\left(\bmod X^{M m \cdot m^{s-1}}\right), \\
((X+\delta)(F+\varphi))^{m} & =(X+\delta)^{m}\left(F^{m}+o(1)\right) \\
& =(X F)^{m}+o(1) \quad\left(\bmod X^{M \cdot m^{s-1}}\right) .
\end{aligned}
$$

From $\left(\mathrm{A}_{s-1}\right)$ we get

$$
(X+\delta)(F+\varphi)=X F+o(1) \quad\left(\bmod X^{M m}\right),
$$

and therefore (by $\left.\left(A_{1}\right)\right)$

$$
\varphi \longrightarrow 0 \quad\left(\bmod X^{M}\right) .
$$

This completes the proof of $\left(\mathrm{A}_{s}\right)$ and Lemma 6 .

Q.E.D.

LEMMA 7.

a) Let $f: \boldsymbol{R} \rightarrow \boldsymbol{C}$ be a function, flat on the closed set $P$ and smooth on $\boldsymbol{R} \backslash P$ (and therefore continuous). If $f^{m} \in C^{\infty}$ and $f \in C^{n-1} \backslash C^{n}$, for some natural numbers $m$ and $n$, then there is $a p \in P$ and a sequence of intervals $I_{k}=\left[a_{k}, b_{k}\right]$ with $p<$ $a_{k}<b_{k}$ (or $\left[b_{k}, a_{k}\right]$ with $b_{k}<a_{k}<p$ ), $I_{k} \subset \boldsymbol{R} \backslash P, b_{k} \rightarrow p$, and two positive constants $c$ and $K$, such that

$$
\begin{aligned}
& \left|f^{(n)}\left(b_{k}\right)\right| \geqq c, \\
& \sup _{I_{k}}|f|=K\left|I_{k}\right|^{n}, \\
& \lim _{k \rightarrow \infty} \frac{\inf _{I_{k}}|f|}{\sup _{I_{k}}|f|}<1 .
\end{aligned}
$$

b) Moreover suppose that $g: \boldsymbol{R} \rightarrow \boldsymbol{C}$ is a smooth function such that $f g$ is smooth. Then one has for all $s, \lambda \in N \cup\{0\}$

$$
\sup _{I_{k}}\left|g^{(s)}\right|=o\left(\left|I_{k}\right|^{\lambda}\right), \quad \text { as } \quad k \rightarrow \infty .
$$

Remark. (14) follows without the hypothesis $f^{m} \in C^{\infty}$.

Proof. a) Let us first prove the existence of a sequence $b_{k}$ verifying (14), $b_{k} \notin P$. We consider two possibilities, supposing first that $f^{(n)}$ exists everywhere. $f \notin C^{n}$, so $f^{(n)}$ is non-continuous at some $p \in P$. By Lemma $2, f^{(n)}(p)=0$, thus $\lim \sup _{x \rightarrow 0}\left|f^{(n)}(x)\right|>0$, which proves the existence of the sequence $b_{k}\left(b_{k} \notin P\right.$ follows from Lemma 2). Next we suppose that $f^{(n)}$ is not defined at a $p \in P$, say $p=0$. Because of $f^{(n-1)}(0)=0$ one has

$$
\limsup _{x \rightarrow 0}\left|f^{(n-1)}(x) / x\right| \geqq 4 c>0 ;
$$

so there is a sequence $x_{k} \rightarrow 0$, say $x_{k}>0$, such that $\left|f^{(n-1)}\left(x_{k}\right)\right| \geqq 2 c x_{k}$. If $\xi_{k}$ is 
defined by $\xi_{k}=\sup \left(P \cap\left[0, x_{k}\right]\right)$, we have $\xi_{k} \in P$ and, by Lemma $2,0 \leqq \xi_{k}<x_{k}$, $f^{(n-1)}\left(\xi_{k}\right)=0$. Replacing the sequence by a subsequence and multiplying $f$ by an appropriate constant if necessary, we may suppose that $\Re f^{(n-1)}\left(x_{k}\right) \geqq c x_{k}$, where $\Re$ stands for "real part of". Then there is $b_{k}, \xi_{k}<b_{k}<x_{k}$, such that

$$
\begin{aligned}
c x_{k} \leqq \Re f^{(n-1)}\left(x_{k}\right) & =\Re f^{(n-1)}\left(x_{k}\right)-\Re f^{(n-1)}\left(\xi_{k}\right) \\
& =\left(x_{k}-\xi_{k}\right) \Re f^{(n)}\left(b_{k}\right) \leqq x_{k} \Re f^{(n)}\left(b_{k}\right),
\end{aligned}
$$

which implies (14). To prove (15), we shall suppose for simplicity that $p=0$, $b_{k}>0, c=4$ and that $\Re f^{(n)}\left(b_{k}\right) \geqq 2$. The continuity of $f^{(n)}$ in the open set $\boldsymbol{R} \backslash P$, implies that there is $d_{k}, 0<d_{k}<b_{k}$, such that $\left[d_{k}, b_{k}\right] \subset \boldsymbol{R} \backslash P, \Re f^{(n)}(x) \geqq 1$ in $\left[d_{k}, b_{k}\right]$, and hence

$$
\sup _{\left[d_{k}, b_{k}\right]}|f| \geqq \sup _{\left[d_{k}, b_{k}\right]}|\Re f| \geqq K\left(b_{k}-d_{k}\right)^{n},
$$

where $K>0$ is the constant $K_{n}$ of Lemma 3 . Set $\xi_{k}=\sup \left(P \cap\left[0, b_{k}\right]\right)$; by Lemma 4 there is a positive constant $C$, independent of $k$, such that $|f(x)| \leqq$ $C\left(x-\xi_{k}\right)^{n+1} \leqq C b_{k}\left(b_{k}-\xi_{k}\right)^{n} \leqq K / 2\left(b_{k}-\xi_{k}\right)^{n}$, for $\xi_{k} \leqq x \leqq b_{k}$ and $k$ large enough. The function $M$, defined for $\xi_{k} \leqq y \leqq b_{k}$ by

$$
M(y)=\sup _{y \leqq x \leqq b_{k}}|f(x)|\left(b_{k}-y\right)^{-n},
$$

is continuous and satisfies the inequalities $M\left(d_{k}\right) \geqq K, M\left(\xi_{k}\right) \leqq K / 2$, thus $M\left(a_{k}\right)$ $=K$ for an $a_{k}$ with $\xi_{k}<a_{k} \leqq d_{k}$; this ends the proof of (15).

In order to prove (16), we set

$$
\Phi_{k}(x)=\left|I_{k}\right|^{-n} f\left(b_{k}-\left|I_{k}\right| x\right), \quad 0 \leqq x \leqq 1,
$$

and use Lemma 5 on $f^{m}$ :

$$
\Phi_{k}^{m}(x)=\left|I_{k}\right|^{-n m} f^{m}\left(b_{k}-\left|I_{k}\right| x\right)=P_{k}(x)+o(1)
$$

in $\boldsymbol{F}$, with $\operatorname{deg} P_{k}<n m$; we have $\left\|P_{k}\right\|=K^{m}+o(1)$, from (15). By compactness we may then suppose that the sequence $P_{k}$ is convergent, so that

$$
\Phi_{k}^{m}(x)=P(x)+o(1) \quad \text { in } \boldsymbol{F},
$$

with a fixed polynomial $P, \operatorname{deg} P<n m,\|P\|=K^{m}$. Now suppose that (16) does not hold for any subsequence of the $I_{k}$. Then $\inf _{[0,1]}\left|\Phi_{k}\right|=K+o(1)$, hence $\inf _{[0,1]}|P|=\sup _{[0,1]}|P|,|P(x)|$ is constant, and so $P(x)$, being a polynomial, is constant too. Therefore we have in $\boldsymbol{F}$ :

$$
m \Phi_{k}^{\prime}=\frac{\left(\Phi_{k}^{m}\right)^{\prime}}{\Phi_{k}^{m-1}}=\frac{(P+o(1))^{\prime}}{\Phi_{k}^{m-1}}=\frac{o(1)}{\Phi_{k}^{m-1}} .
$$

From this we deduce, by iterated differentiations, that $\Phi_{k}^{(s)}=o(1) \Phi_{k}^{1-s m}, s=$ $1,2,3, \cdots$; in particular $f^{(n)}\left(b_{k}\right)= \pm \Phi_{k}^{(n)}(0) \rightarrow 0$, which contradicts (14). This proves (16). 
b) Suppose that (17) is not true. Then there are integers $s, \lambda \geqq 0$ and real $c_{1}>0$ such that

$$
\sup _{I_{k}}\left|g^{(s)}\right| \geqq c_{1}\left|I_{k}\right|^{\lambda}
$$

taking again a subsequence if necessary. As $g, f g$ and $f^{m}$ are smooth (and $f$ is not smooth at $p) g$ and $f g$ are flat at $p$, by Lemma 1. Let us apply Lemma 5 to the functions $g$ and $g f$ :

$$
\begin{aligned}
& \left|I_{k}\right|^{-s-\lambda} g\left(b_{k}-\left|I_{k}\right| x\right)=G_{k}+o(1), \\
& \left|I_{k}\right|^{-s-\lambda-\lambda}(f g)\left(b_{k}-\left|I_{k}\right| x\right)=H_{k}+o(1)
\end{aligned}
$$

in $\boldsymbol{F}$, where $G_{k}$ and $H_{k}$ are polynomials, $\operatorname{deg} G_{k}<s+\lambda, \operatorname{deg} H_{k}<\dot{n}+s+\lambda,\left\|G_{k}\right\|$ $\gg 1$. From $f^{m} g^{m}=(f g)^{m}$ and (20) we infer

$$
\left(\frac{G_{k}}{\left\|G_{k}\right\|}+o(1)\right)^{m}(P+o(1))=\left(\frac{H_{k}}{\left\|G_{k}\right\|}+o(1)\right)^{m} ;
$$

this implies $\left\|H_{k}\right\| /\left\|G_{k}\right\| \ll 1$. Taking again a subsequence, we find polynomials $G$ and $H$ such that $G \neq 0, \operatorname{deg} G<s+\lambda, \operatorname{deg} H<n+s+\lambda$, and such that $G_{k} /\left\|G_{k}\right\| \|$ $G+o(1), \quad H_{k} /\left\|G_{k}\right\|=H+o(1)$ in $\boldsymbol{F}$; then $(G+o(1))^{m}(P+o(1))=(H+o(1))^{m}$, and hence $P G^{m}=H^{m}$. There is a polynomial $F$ such that $P=F^{m}, H=F G$, $\operatorname{deg} F=(1 / m) \operatorname{deg} P<n$. Set

$$
\varphi_{k}=\Phi_{k}-F
$$

By (21) and (22), we have

$$
(G+o(1))\left(F+\varphi_{k}\right)=F G+o(1)
$$

and by (20)

$$
\left(F+\varphi_{k}\right)^{m}=F^{m}+o(1) .
$$

Taking Taylor series', one has in $\boldsymbol{E}:(G+o(1))\left(F+T \varphi_{k}\right)=G F+o(1)$ and $\left(F+T \varphi_{k}\right)^{m}$ $=F^{m}+o(1)$. By Lemma 6 we obtain $T \varphi_{k} \rightarrow 0$ in $E$, and then $\varphi_{k}^{(n)}(0)=\left(T \varphi_{k}^{(n)}\right)(0)$ $\rightarrow 0$. But $\pm f^{(n)}\left(b_{k}\right)=\Phi_{k}^{(n)}(0)=F^{(n)}(0)+\varphi_{k}^{(n)}(0)=\varphi_{k}^{(n)} \rightarrow 0$ (because $\operatorname{deg} F<n$ ); this contradicts (14). Thus Lemma 7 is proved.

Q.E.D.

Proof of Theorem 1. If $P$ is the set of flatness of $g$ then (by Lemma 1) $f$ is smooth on $\boldsymbol{R} \backslash P$, and by $|f| \leqq c|g|^{\alpha}, f$ is flat on $P$. Thus $f$ is continuous. If $f$ is not smooth, there is a $n \in N$ such that $f \in C^{n-1} \backslash C^{n}$. We apply Lemma 7 , with $s=0, \lambda=n / \alpha$. Then

$$
\begin{aligned}
& \sup _{I_{k}}|f| \gg\left|I_{k}\right|^{n}, \\
& \sup _{I_{k}}|g|=o\left(\left|I_{k}\right|^{n / \alpha}\right),
\end{aligned}
$$

which is inconsistent with $|f| \leqq c|g|^{\alpha}$.

Q.E.D. 


\section{Pseudo-immersions.}

In this section we shall use Theorem 1 to deduce sufficient conditions for the smoothness of $n$ functions $f_{1}, \cdots, f_{n}$.

THEOREM 2. If $m_{1}, m_{2}, \cdots, m_{n}$ and $s_{1}, s_{2}, \cdots, s_{n}$ are natural numbers such that

$$
\text { g.c. d. }\left(m_{\jmath}, 2 s_{\jmath}\right)=1, \quad j=1, \cdots, n \text {, }
$$

and if $f_{1}, \cdots, f_{n}: \boldsymbol{R} \rightarrow \boldsymbol{R}$ are such that

$$
f_{1}^{m_{1}}, \cdots, f_{n}^{m_{n}}, \sum_{1}^{n} f_{\jmath}^{2 s_{\jmath}} \in C^{\infty},
$$

then

$$
f_{1}, \cdots, f_{n} \in C^{\infty} \text {. }
$$

Using the definition of pseudo-immersions we may write Theorem 2 in the following way:

THEOREM $2^{\prime}$. If $m_{1}, \cdots, m_{n}$ and $s_{1}, \cdots, s_{n}$ are natural numbers such that

$$
\text { g.c.d. }\left(m_{\jmath}, 2 s,\right)=1, \quad j=1,2, \cdots, n \text {. }
$$

Then the map

$$
h: \boldsymbol{R}^{n} \longrightarrow \boldsymbol{R}^{n+1}, \quad\left(x_{1}, \cdots, x_{n}\right) \longmapsto\left(x_{1}^{m_{1}}, \cdots, x_{n}^{m}, \sum_{1}^{n} x_{\jmath}^{2 s_{\jmath}}\right)
$$

is a pseudo-immersion.

Condition (26) is clearly necessary because if, say, g.c. d. $\left(m_{1} \cdot 2 s_{1}\right)=p>1$, we may choose $f_{2}=\cdots=f_{n}=0, f_{1}(t)=|t|$ if $p$ is even, and $f_{1}(t)=t^{1 / p}$ if $p$ is odd. Then $f \notin C^{\infty}$, but $h \circ f \in C^{\infty}$. Essential tools for the proof of Theorem 2 are rational representations

$$
x_{j}=\frac{P\left(x_{1}^{m_{1}}, \cdots, x_{n}^{m_{n}}, \sum_{1}^{n} x_{J}^{2 s_{j}}\right)}{Q\left(x_{1}^{m_{1}}, \cdots, x_{n}^{m_{n}}, \sum_{1}^{n} x_{j}^{2 s_{j}}\right)},
$$

where roots of the denominator are well controlled. We set

$$
X=\left(X_{1}, \cdots, X_{n}\right), \quad U=\left(U_{1}, \cdots, U_{n} ; U_{0}\right)
$$

for indeterminates. A substitution by real or complex numbers is given by corresponding small letters. We shall write $X^{m}$ for $\left(X_{1}^{m_{1}}, \cdots, X_{n}^{m_{n}}\right)$ and $\Sigma X^{2 s}$ for $\Sigma_{1}^{n} X_{j}^{2 s}$, with analogous abbreviations for substitutions.

LEMMA 8.

a) The ring of polynomials $\boldsymbol{C}[X]$ is an integral extension of the subring $\boldsymbol{C}\left[X^{m}, \Sigma X^{2 s}\right]$, finitely generated as a module over $\boldsymbol{C}\left[X^{m}, \Sigma X^{2 s}\right]$. 
b) The kernel of the ring homomorphism

$$
H: C[U] \longrightarrow C[X], \quad p(U) \longmapsto p\left(X^{m}, \Sigma X^{2 s}\right)
$$

is a principal prime ideal $(N)$ generated by an irreducible polynomial $N(U) \in C^{\prime}\left[l^{\prime}\right]$. and

$$
\operatorname{grad} N\left(x^{m}, \Sigma x^{2 s}\right) \neq 0 \quad \text { if } \quad x \in \boldsymbol{R}^{n}, x_{1} x_{2} \cdots x_{n} \neq 0
$$

Proof.

a) This follows from the fact that the generators $X_{1}, \cdots, X_{n}$ of $C[X]$ are integral over $\boldsymbol{C}\left[X^{m}, \Sigma X^{2 s}\right]$ (see $[Z S]$, p. 254).

b) We have

$$
\boldsymbol{C}(X)=\boldsymbol{C}\left(X^{m}, \Sigma X^{2 s}\right),
$$

for the degree of $X$, on $\boldsymbol{C}\left(X^{m}\right)$ is $m_{\jmath}$, so the degree of $\boldsymbol{C}(X)$ over $\boldsymbol{C}\left(X^{m}\right)$ is at most $m_{1} m_{2} \cdots m_{n}$. On the other hand, the field $\boldsymbol{C}(X)$ admits the group $\Gamma$ of $m_{1} m_{2} \cdots m_{n}$ automorphisms over $\boldsymbol{C}\left(X^{m}\right)$

$$
X \longmapsto \zeta X, \quad \zeta=\left(\zeta_{1}, \cdots, \zeta_{n}\right)
$$

i.e. $X_{1} \mapsto \zeta_{1} X_{1}, \cdots, X_{n} \mapsto \zeta_{n} X_{n}$, where $\zeta_{3}$ is a $m_{\jmath}^{t h}$ root of unity. The identity corresponds to $(1, \cdots, 1)=1$. Thus $C(X)$ is a Galois extension of $\boldsymbol{C}\left(X^{m}\right)$, with the Galois group $\Gamma$. Because of $(26),\left(\zeta_{j} X_{j}\right)^{2 s_{j}}=X_{\jmath}^{2 s}$ if $\zeta_{j}=1, \Sigma(\zeta X)^{2 s} \neq \Sigma X^{2 s}$ if $\zeta \neq 1$. By Galois theory (see [ZS], p. 80) this implies $\boldsymbol{C}(X)=\boldsymbol{C}\left(X^{m}, \Sigma X^{2 s}\right)$.

The monic irreducible polynomial of $\Sigma X^{2 s}$ over $\boldsymbol{C}\left(X^{m}\right)$ is given by

$$
N\left(X^{m} ; U_{0}\right)=\prod_{\zeta}\left(U_{0}-\Sigma(\zeta X)^{2 s}\right) \in C\left[X^{m} ; U_{0}\right]
$$

with

We have

$$
N(U) \equiv \boldsymbol{C}[U] .
$$

$$
H N=N\left(X^{m}, \Sigma X^{2 s}\right)=0 .
$$

If $p(U) \in C[U]$ and $p\left(X^{m}, \Sigma X^{2 s}\right)=0$, then $p\left(X^{m} ; U_{0}\right)$ is a multiple of $N\left(X^{m} ; U_{0}\right)$ in $C\left[X^{m}\right]\left[U_{0}\right]$ because $N$ is monic (for $U_{0}$ ); thus $N$ generates the kernel of $H$. But the image of $H$ is an integral domain, the kernel $(N)$ is prime, and $N$ is irreducible. For the last assertion of the lemma, we observe that

$$
\begin{gathered}
\frac{\partial N}{\partial U_{0}}\left(X^{m}, \Sigma X^{2 s}\right)=\prod_{\zeta \neq 1}\left(\Sigma X^{2 s}-\Sigma(\zeta X)^{2 s}\right), \\
m_{\jmath} X_{\jmath}^{m_{j}-1} \frac{\partial N}{\partial U_{\jmath}}\left(X^{m} ; \Sigma X^{2 s}\right)=-2 s_{\jmath} X_{\jmath}^{2 s_{j}-1} \prod_{\zeta \neq 1}\left(\Sigma X^{2 s}-\Sigma(\zeta X)^{2 s}\right), \quad j=1, \cdots, n .
\end{gathered}
$$

We replace $X$ by real non-zero variables $x_{1}, \cdots, x_{n}$. If $\zeta \neq 1$, say $\zeta_{1} \neq 1$, we have

$$
\Re\left(\Sigma x^{2 s}-\Sigma(\zeta x)^{2 s}\right) \geqq x_{1}^{2 s_{1}} \Re\left(1-\zeta_{1}^{2 s}\right)>0
$$


by (26); hence $\Sigma x^{2 s}-\Sigma(\zeta x)^{2 s} \neq 0$. This proves (27).

Q.E.D.

We shall write

$$
\Omega=\left\{x \in \boldsymbol{R}^{n} \mid x_{1} x_{2} \cdots x_{n} \neq 0\right\} .
$$

Consider the algebraic set

$$
V=\left\{u \in \boldsymbol{C}^{n+1} \mid N(u)=0\right\} \subset \boldsymbol{C}^{n+1} .
$$

$V$ is irreducible and its ring of regular functions is $C[U] / N \cong C\left[X^{m}, \Sigma X^{2 s}\right]$. The points $\left(x^{m}, \Sigma x^{2 s}\right)$ are in $V$ and (27) shows that if $x \in \Omega$, then $\left(x^{m}, \Sigma x^{2 s}\right)$ is a regular point of $V$ (see [S], p. 71-78). This implies that the local ring of $V$ at $\left(x^{m}, \Sigma x^{2 s}\right)$ is integrally closed ([S], p. 109-110). This local ring is the ring of all quotients

$$
\frac{P\left(X^{m}, \Sigma X^{2 s}\right)}{Q\left(X^{m}, \Sigma X^{2 s}\right)}
$$

where $P$ and $Q$ are polynomials with $Q\left(x^{m}, \Sigma x^{2 s}\right) \neq 0$.

LEMma 9 (see [ZS], p. 260). Let $A$ be an integral domain, $K$ its field of fractions, and $A^{\prime}$ the integral closure of $A$ in $K$. We suppose that $A^{\prime}$ is a finite A-module. Set

$$
C=\left\{a \in A \mid a A^{\prime} \subset A\right\}
$$

( $C$ is the conductor of $A$ in $A^{\prime}$ ). The following equivalence holds If $S \subset A$ is a multiplicatively closed set, the ring of fractions $A_{S}:=\{a / s \mid a \in A, s \in S\}$ is integrally closed in $K$ if and only of $C \cap S$ is non-empty.

We apply this lemma to $A=\boldsymbol{C}\left[X^{m}, \Sigma X^{2 s}\right], A^{\prime}=\boldsymbol{C}[X], K=\boldsymbol{C}(X)$. The assumptions concerning $A$ and $A^{\prime}$ are satisfied by Lemma 8(a) and by the fact that $\boldsymbol{C}[X]$ is integrally closed (cf. $[Z \mathrm{~S}]$, p. 261, ex. 1). For $x \in \Omega$, set

$$
S_{x}=\left\{Q \in C\left[X^{m}, \Sigma X^{2 s}\right] \mid Q\left(x^{m}, \Sigma x^{2 s}\right) \neq 0\right\} .
$$

$A_{S_{x}}$ is the local ring of $V$ at $\left(x^{m}, \Sigma x^{2 s}\right)$. We have already remarked that it is integrally closed, thus $S_{x} \cap C$ in not empty. For each $x \in \Omega$, we choose $Q_{x} \in$ $S_{x} \cap C$, and we write $I$ for the ideal generated in $A$ by all the $Q_{x}, x \in \Omega$. As $C$ is an ideal in $A$ one has $I \subset C$, thus $I A^{\prime} \subset A$. $A$ is noetherian, $I$ is therefore generated by finitely many polynomials $P_{1}, \cdots, P_{k}$. As $Q_{x} \in I$ for all $x \in \Omega$, one of the $P$, does not vanish at $x$. Therefore the polynomial $\Delta$, defined by

$$
\Delta=\sum_{1}^{k} P_{j} \bar{P}_{j} \epsilon=I
$$

(the bar means that each coefficient is replaced by its complex conjugate) does not vanish at $\Omega$, and $\Delta A^{\prime} \subset A$. The coefficients of $\Delta$ being real, we finally obtain

$$
\Delta\left(X^{m}, \Sigma X^{2 s}\right) \boldsymbol{R}[X] \subset \boldsymbol{R}\left[X^{m}, \Sigma X^{2 s}\right], \quad \Delta\left(x^{m}, \Sigma x^{2 s}\right) \neq 0 \quad \text { if } x \in \Omega .
$$


The next result is due to $S$. Lojasiewicz ([L], p. 124 ; see also [M], p. 59, for a proof):

LEMMA 10. Let $\varphi: U \rightarrow \boldsymbol{R}$ be a real-analytical function on the open set $U \subset \boldsymbol{R}^{n}$, and set $Z=\varphi^{-1}(0)$. For each compact $K \subset U$ there exist positive numbers $c=c_{K}$, $\alpha=\alpha_{K}$ such that

$$
|\varphi(x)| \geqq c d(x, Z)^{\alpha}, \quad \text { for all } x \in K,
$$

where $d(x, Z)$ stands for the distance from $x$ to $Z$.

We apply this lemma to $\varphi(x)=\Delta\left(x^{m}, \Sigma x^{2 s}\right)$. We have $Z \subset\left\{x \mid x_{1} x_{2} \cdots x_{n}=0\right\}$ since $\varphi(x) \neq 0$ for $x \in \Omega$. If $\left|x_{j}\right| \leqq M, j=1,2, \cdots, n$ then

$$
d(x, Z) \geqq \min \left(\left|x_{1}\right|, \cdots,\left|x_{n}\right|\right) \geqq \frac{\left|x_{1} \cdots x_{n}\right|}{M^{n-1}} .
$$

Therefore

$$
\left|\Delta\left(x^{m}, \Sigma x^{2 s}\right)\right| \gg\left|x_{1} \cdots x_{n}\right|^{\alpha},
$$

for $x$ bounded.

LEMMA 11. If the assumptions are as in Theorem 2, and if $a_{1}, \cdots, a_{n} \in \boldsymbol{N}$, then

$$
f_{1}^{a_{1}} f_{2}^{a_{2}} \cdots f_{n}^{a_{n}} \in C^{\infty}
$$

Proof. By (29) we have

$$
X_{1}^{a_{1}} \cdots X_{n}^{a}{ }_{n} \Delta\left(X^{m}, \Sigma X^{2 s}\right)=P\left(X^{m}, \Sigma X^{2 s}\right) .
$$

where $P$ is a real polynomial. Thus

$$
f_{1}^{a_{1}} \cdots f_{n}^{a_{n}} \Delta\left(f^{m}, \Sigma f^{2 s}\right)=P\left(f^{m}, \Sigma f^{2 s}\right),
$$

where $\Delta\left(f^{m}, \Sigma f^{2 s}\right)$ and $P\left(f^{m}, \Sigma f^{2 s}\right)$ are smooth functions. The $f$, are continuous and therefore locally bounded. By (30) we thus obtain

$$
\left|\Delta\left(f^{m}, \Sigma f^{2 s}\right)\right| \gg\left|f_{1} \cdots f_{n}\right|^{\alpha} \gg\left|f_{1}^{a_{1}} \cdots f_{n}^{a_{n}}\right|^{\beta}, \quad \beta=\alpha / \inf a_{\jmath},
$$

in bounded intervals. Then $f_{1}^{a_{1}} \cdots f_{n}^{a} n \in C^{\infty}$ follows from Theorem 1. Q.E.D.

Proof of Theorem 2. The theorem is true for $n=1$, by the corollary to Theorem 1. We shall proceed by induction. By the assumptions of the theorem, we have

$$
\left(f_{1}^{2 s_{1}}+\cdots+f_{n}^{2 s_{n}}\right) f_{2}^{2 m_{2}} \cdots f_{n}^{2 m_{n}} \in C^{\infty} .
$$

By Lemma 11, we may drop the first product, therefore 


$$
\sum_{j=2}^{n}\left(f_{j}^{s_{j}}\left(f_{2}^{m_{2}} \cdots f_{n}^{m_{n}}\right)\right)^{2} \in C^{\infty}
$$

Since

$$
\left(f_{j}^{s_{\jmath}}\left(f_{2}^{m_{2}} \cdots f_{n}^{m_{n}}\right)\right)^{m_{\jmath}} \in C^{\infty}, \quad j=2, \cdots, n,
$$

we may use the induction hypthesis with $s_{2}=\cdots=s_{n}=1$ to obtain

$$
f_{j}^{s_{j}}\left(f_{2}^{m_{2}} \cdots f_{n}^{m_{n}}\right) \in C^{\infty}, \quad \jmath=2, \cdots, n .
$$

Put $S=\max s_{\jmath}, M=\max m_{\jmath}$. We shall show that if $b_{2}, \cdots, b_{n}>S M+(n-1) M^{2}$ then

$$
f_{2}^{b_{2}} \ldots f_{n}^{b_{n}} \in C^{\infty} \text {. }
$$

and therefore, by the corollary to Theorem 1 ,

$$
f_{2}^{a_{2}} f_{3}^{a_{3}} \cdots f_{n}^{a_{n}} \in C^{\infty}, \quad \text { if } \quad a_{2}, \cdots, a_{n} \geqq 1 .
$$

Since $\mathbf{s}_{\jmath}, m_{\jmath}$ are coprime, one can find $\nu_{j} \in N, 0 \leqq \nu_{\jmath} \leqq m_{\jmath}-1$, such that

$$
s_{j} \nu_{\jmath} \equiv b_{\jmath} \quad\left(\bmod m_{\jmath}\right), \quad \jmath=2, \cdots, n .
$$

By (31) we obtain

$$
\prod_{j=2}^{n} f_{j}^{s j^{\nu} \nu}\left(f_{2}^{m_{2}} \cdots f_{n}^{m_{n}}\right)^{\nu} \jmath \in C^{\infty}
$$

The contribution of $f_{2}$ to this product is

$$
f_{2}^{s_{2} \nu_{2}} f_{2}^{m_{2}\left(\nu_{2}+\cdots+\nu_{n}\right)}=f_{2}^{q}
$$

where $q \equiv b_{2}\left(\bmod m_{2}\right)$, and $q \leqq S M+(n-1) M^{2}<b_{2}$. Therefore, if we multiply by an appropriate power of $f_{2}^{m_{2}}, f_{2}$ will have the desired exponent $b_{2}$. We proceed in the same way with the other $f_{\jmath}$. This proves (32), and thus (33).

We start the whole thing all over again, noting that

$$
\left(f_{1}^{2 s_{1}}+\cdots+f_{n}^{2 s_{n}}\right) f_{3}^{2 m_{3}} \cdots f_{n}^{2 m_{n}} \in C^{\infty}
$$

and therefore

$$
\left(f_{3}^{2 s_{3}}+\cdots+f_{n}^{2 s_{n}}\right) f_{3}^{2 m_{3}} \cdots f_{n}^{2 m_{n}} 巨 C^{\infty},
$$

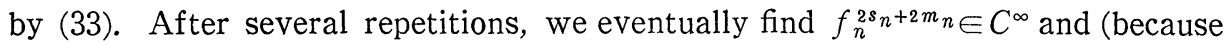
$\left.f_{n}^{m} \subseteq C^{\infty}\right) f_{n} \subseteq C^{\infty}$.

Q.E.D.

Let us change the assumptions in Theorem 2 .

$$
f_{1}^{m_{1}}, \cdots, f_{n}^{m_{n}}, \sum_{1}^{N} f_{j}^{s_{\jmath}} \in C^{\infty}, \quad \text { g.c.d. }\left(m_{\jmath}, s_{\jmath}\right)=1, \quad j=1,2, \cdots, n .
$$

The exponents in the sum may be odd. It is easy to prove a lemma analogous to Lemma 8, except for (27), which is wrong in general. However, let us consider a special case: 


$$
n=2, \quad s_{1}=s_{2}=1, \quad \text { g.c.d. }\left(m_{1}, m_{2}\right)=1 \text {. }
$$

In order to prove (27), we have to show that if $x, y$ are real numbers, $\zeta$, a $m_{j}$-th root of unity, $j=1,2,\left(\zeta_{1}, \zeta_{2}\right) \neq(1,1)$, and if $x+y=\zeta_{1} x+\zeta_{2} y$, then $x y=0$. If $\zeta_{1}=1$, then $\zeta_{2} \neq 1$ and thus $y=0$; we have the same result if $\zeta_{1}=1$. Now, let us suppose that $\zeta_{1} \neq 1, \zeta_{2} \neq 1$, and set $\zeta_{1}=\exp \left(2 \pi i a / m_{1}\right), 0<a<m_{1}, \zeta_{2}=\exp \left(2 \pi i b / m_{2}\right)$, $0<b<m_{2}$. We obtain the following linear system

$$
\begin{aligned}
& x\left(1-\zeta_{1}\right)+y\left(1-\zeta_{2}\right)=0 \\
& x\left(1-\bar{\zeta}_{1}\right)+y\left(1-\bar{\zeta}_{2}\right)=0
\end{aligned}
$$

which must be singular if there were to be a solution such that $x y \neq 0$ :

$$
\frac{1-\zeta_{1}}{1-\bar{\zeta}_{1}}=\frac{1-\zeta_{2}}{1-\bar{\zeta}_{2}}
$$

and by an easy calculation

$$
\exp \left(2 \pi i \frac{a}{m_{1}}\right)=\exp \left(2 \pi i \frac{b}{m_{2}}\right) .
$$

This is not possible under our assumptions. Now we can go on as in the proof of Theorem 2 and show that $f_{1}$ and $f_{2}$ are smooth. Somewhat more generally we get:

THEOREM 3. Let $m, n, r, s$ be natural numbers such that

$$
\text { g.c.d. }(m, n)=\text { g.c.d. }(m, r)=\text { g.c.d. }(n, s)=1 \text {, }
$$

and let $f, g: \boldsymbol{R} \rightarrow \boldsymbol{R}$ be functions such that

$$
f^{r}+g^{s}, f^{m}, g^{n} \in C^{\infty} .
$$

Then $f, g \in C^{\infty}$.

Proof. We just did the proof in the special case $r=s=1$. By the hypothesis, $\left(f^{r}\right)^{m},\left(g^{s}\right)^{n}$ and $f^{r}+g^{s}$ are smooth, therefore $f^{r}$ and $g^{s}$ too are smooth, and so are $f$ and $g$ by the corollary to Theorem 1 .

The conditions g.c.d. $(m, r)=$ g.c.d. $(n, s)=1$ are necessary, as is shown by the counterexamples given just after Theorem $2^{\prime}$. If $r$ or $s$ is odd, the condition g.c.d. $(m, n)=1$ is necessary too. In fact, if $q=$ g.c.d. $(m, n)>1$, then we define $f(t)=|t|^{s}, g(t)=-|t|^{r}$ if $q$ is even, and $f(t)=t^{s / q}, g(t)=-t^{r / q}$ if $q$ is odd and $s$ is odd, say. Combining these remarks with Theorem 3 and Theorem $2^{\prime}$, we obtain:

THEOREM $3^{\prime}$. The map

$$
\boldsymbol{R}^{2} \longrightarrow \boldsymbol{R}^{3}, \quad(x, y) \longmapsto\left(x^{r}+y^{s}, x^{m}, y^{n}\right),
$$

is a pseudo-immersion if and only if 
a) g.c.d. $(r, m)=$ g.c.d. $(s, n)=1$;

b) $r, s$ are even or g.c.d. $(m, n)=1$.

\section{Examples and counterexampls.}

Pseudo-immersivity being a local property, we shall consider smooth germs

$$
h:\left(\boldsymbol{R}^{n}, 0\right) \longrightarrow\left(\boldsymbol{R}^{m}, 0\right) .
$$

The family of all such germs will be denoted by $\Gamma_{m, n}, h \in \Gamma_{m, n}$ is pseudoimmersive if $h$ is represented by a pseudo-immersion. We shall write $\phi_{m, n}$ for the family of all pseudo-immersive $h \in \Gamma_{m, n}$. In this section we shall answer some questions that arise quite naturally in studying pseudo-immersions.

a) In [JP1] we have determined all the germs $h \in \phi_{m, 1}$, and proved that the pseudo-immersivity of $h \in \Gamma_{m, 1}$, depends only on the Taylor series Th. Is this still true for $\Gamma_{m, n}$ with an arbitrary $n$ ? The (negative) answer is provided by the following example. Set

$$
g(x, y)=\left(x^{2}, x^{3}, y\right) .
$$

$g$ is the cartesian product of the (pseudo-immersive) identity and the map $x \mapsto$ $\left(x^{2}, x^{3}\right)$ which is pseudo-immersive by the corollary to Theorem 1 ; hence $g \in \phi_{3,2}$. Let $\omega: \boldsymbol{R} \rightarrow \boldsymbol{R}$ be defined by

$$
\begin{aligned}
& \omega(y)=\exp \left(-2 y^{-2}\right) \sin ^{2}(1 / y), \quad \text { if } y \neq 0, \\
& \omega(0)=0 .
\end{aligned}
$$

With this we now define a germ

$$
h(x, y)=\left(x^{2}, x^{3}-x \omega(y), y\right)
$$

which has the same Taylor expansion as $g$ but is not pseudo-immersive. Indeed, $T h=T g$ follows from the flatness of $\omega$ at 0 . And if $f(t)=(\sqrt{\omega(t)}, t)=$ $\left(\exp \left(-t^{-2}\right)|\sin (1 / t)|, t\right)$, then $f$ is continuous, $f \notin C^{1}$, but $h \circ f(t)=(\omega(t), 0, t)$, and therefore $h \circ f \in C^{\infty}$. Thus we have proved

THEOREM 4. There exist $g, h \in \Gamma_{m, n}$ such that $T g=T h, g \in \phi_{m n}, h \notin \phi_{m, n}$.

In our counterexample, $h$ is neither analytic not injective, which leads us to ask the following questions:

Let $g, h \in \Gamma_{m, n}, g \in \psi_{m, n}, T g=T h$. Does any of the three conditions listed below imply that $h$ is pseudo-immersive?

(i) $h$ is a polynomial;

(ii) $h$ is analytic;

(iii) $h$ is injective.

Obviously (i) implies (ii). Also (ii) implies (iii), under our assumptions, but this 
is much less obvious.

b) In [JP2] we have shown that a pseudo-immersive germ is almost injective i.e. if $x_{p}, y_{p} \rightarrow 0, h\left(x_{p}\right)=h\left(y_{p}\right)$, then $\left\|\left(x_{p}-y_{p}\right)\right\| \ll\left\|x_{p}\right\|^{\alpha}+\left\|y_{p}\right\|^{\alpha}$ for all $\alpha>0$. But a pseudo-immersive germ may be non-injective as is shown in the following theorem.

THEOREM 5. Set

$$
\begin{aligned}
& h(x, y)=\left(x^{2}, x^{3}-x e^{-1 /|y|}, y\right) \quad \text { if } y \neq 0, \\
& h(x, 0)=\left(x^{2}, x^{3}, 0\right) .
\end{aligned}
$$

Then $h$ represents a pseudo-immersive non-injective germ. The non-injectivity follows from $h\left(e^{-1 / 2|t|}, t\right)=h\left(-e^{-1 / 2|t|}, t\right)$.

Proof. $h$ is immersive except at $(0,0)$; we omit the proof which is straightforward. Let $f: \boldsymbol{R} \rightarrow \boldsymbol{R}^{2}$ be a continuous function such that $h \circ f$ is smooth and set $f(t)=(x(t), y(t))$. Then obviously $y \in C^{\infty}$. By the immersivity of $h$ (except at the origin), we know that $f$ is smooth except possibly at the zeros of $y$. Define $\omega$ by $\omega(u)=\exp (-1 /|u|)$ for $u \neq 0, \omega(0)=0$. If $y\left(t_{0}\right)=0$, then $\omega \circ y$ is flat at $t_{0}$. If $x$ is not flat at $t_{0}$, then $x^{2}-\omega \circ y$ is not flat either and $x$ is smooth at $t_{0}$ by Lemma 1 , because $x^{2}-\omega^{\circ} y, x\left(x^{2}-\omega^{\circ} y\right), x^{2}$ are smooth. Thus $f$ can be nonsmooth only at the points of flatness of $x$. We now apply Lemma 7, with $x$ for $f$, and $g=x^{2}-\omega \circ y, s=0, \lambda=2 n, m=2$. If $f \in C^{n-1} \backslash C^{n}$, we have by (15), (16) and (17):

$$
\begin{gathered}
\sup _{I_{k}}(\omega \circ y) \sim K^{2}\left|I_{k}\right|^{2 n}, \quad \text { for } \quad k \rightarrow \infty, \\
\lim _{k \rightarrow \infty} \frac{\inf _{I_{k}}(\omega \circ y)}{\sup _{I_{k}}(\omega \circ y)}<1 .
\end{gathered}
$$

Therefore there are two constants $B>A>0$ and sequences $t_{k}, s_{k} \in I_{k}$ with $\omega\left(y\left(t_{k}\right)\right)$ $=B\left|I_{k}\right|^{2 n},\left|\omega\left(y\left(s_{k}\right)\right)\right|=A\left|I_{k}\right|^{2 n}$, and $y\left(s_{k}\right)>0, y\left(t_{k}\right)>0$, say. Then

$$
y\left(t_{k}\right)-y\left(s_{k}\right)=y\left(t_{k}\right) y\left(s_{k}\right)\left(\frac{1}{y\left(s_{k}\right)}-\frac{1}{y\left(t_{k}\right)}\right) \sim\left(2 n \log \left|I_{k}\right|\right)^{-2} \log \frac{B}{A},
$$

because $\omega(y)=\exp (-1 / y)$ for $y>0$. On the other hand, since $y$ is smooth and $t_{k}, s_{k} \in I_{k}$, we have $y\left(t_{k}\right)-y\left(s_{k}\right) \ll\left|I_{k}\right|$. But $u=o\left((\log u)^{-2}\right)$ for $u \rightarrow 0$, so there is a contradiction.

Q.E.D.

c) If both mappings $h_{1}: \boldsymbol{R}^{p} \rightarrow \boldsymbol{R}^{r}$ and $h^{2}: \boldsymbol{R}^{q} \rightarrow \boldsymbol{R}^{s}$ are pseudo-immersions, then the cartesian product $h_{1} \times h_{2}: \boldsymbol{R}^{p+q} \rightarrow \boldsymbol{R}^{r+s}$ is a pseudo-immersion too, and similarly for the germs. We call the cartesian product of two germs reducible. More generally, we say that $h \in \Gamma_{m, n}$ is reducible if we can find $h_{1} \in \Gamma_{p, r}, h_{2} \in$ $\Gamma_{q, s}$, with $m=p+q, n=r+s, p, q, r, s \geqq 1$, and germs of diffeomorphisms $S, T$ such that the diagram 


$$
\begin{array}{ccc}
\left(\boldsymbol{R}^{n}, 0\right) & h \\
S \uparrow & \left(\boldsymbol{R}^{m}, 0\right) \\
\left(\boldsymbol{R}^{r}, 0\right) \times\left(\boldsymbol{R}^{s}, 0\right) & \stackrel{\left(h_{1}, h_{2}\right)}{\longrightarrow}\left(\boldsymbol{R}^{p}, 0\right) \times\left(\boldsymbol{R}^{q}, 0\right)
\end{array}
$$

is commutative; $h$ is pseudo-immersive if and only if $h_{1}$ and $h_{2}$ are pseudoimmersive. If $h \in \psi_{n, n}, n \geqq 2$, then $h$ is diffeomorphic, and therefore equivalent to the identity on $\left(\boldsymbol{R}^{n}, 0\right)$ and, consequently, reducible; if $n>m$, then $\psi_{m, n}$ is empty (cf. [JP2]). But for other dimensions irreducible pseud-immersive germs do exist :

THEOREM 6. If $n<m$, there is an irreducible germ in $\phi_{m, n}$.

Proof. For $k \geqq 1$ set

$$
\varphi_{k}\left(x_{1}, \cdots, x_{k}\right)=\left(x_{1}^{3}, \cdots, x_{k}^{3}, \sum_{1}^{k} x_{\jmath}^{2}\right) .
$$

By Theorem 2', $\varphi_{k}$ is a pseudo-immersion. Thus $h(x)=\varphi_{m-1} \circ \varphi_{m-2} \circ \cdots \circ \varphi_{n}$ is a pseudo-immersion too. It is evident that

$$
\varphi_{k}(x)=\|x\|^{2}((0, \cdots, 0,1)+o(1)),
$$

as $x \rightarrow 0$, and therefore

$$
h(x)=\|x\|^{Q}(e+o(1)) .
$$

where $Q=2^{m-n}, e=(0, \cdots, 0,1) \in \boldsymbol{R}^{m}$. Suppose now that $h$ is reducible, as in the diagram (35), and that $T^{\prime}(0)=L$. Then

$$
\left(h_{1}(u), 0\right)=T \circ h \circ S(u, 0)=T\left(\|S(u, 0)\|^{Q}(e+o(1))\right)=\|S(u, 0)\|^{Q}(L e+o(1)),
$$

as $u \rightarrow 0$. Thus

$$
L e=\left(\lim _{u \rightarrow 0} h_{1}(u)\|S(u, 0)\|^{-Q}, 0\right) .
$$

In the same way

$$
L e=\left(0, \lim _{v \rightarrow 0} h_{2}(v)\|S(0, v)\|^{-Q}\right) .
$$

Therefore $L e=0$, which contradicts the inversibility of $L$.

Q.E.D.

\section{Differentiability of quotients of smooth functions.}

It is easy to see that in Theorem 1 none of the conditions $g \in C^{\infty}, f g \in C^{\infty}$. $|f| \leqq|g|^{\alpha}$ may be omitted without adequate replacement. That the condition $f^{m} \in C^{\infty}$ cannot be suppressed either is part of the following theorem.

THEOREM 7. Let $f, g: \boldsymbol{R} \rightarrow \boldsymbol{C}$ be two functions and $\alpha$ a positive constant; suppose that 
(a) $g, f g \in C^{\infty}$;

(b) $|f(x)| \leqq|g(x)|^{\alpha}$ for all real $x$.

Then

and

$$
f \in C^{[2 \alpha]} \text {, if } f \text { is real, }
$$

$$
f \in C^{[\alpha]}, \quad \text { if } f \text { is complex. }
$$

This result is best possible: there are real functions $f, g$ satisfying (a) and (b), with $f \notin C^{[2 \alpha]+1}$; there are complex functions $f, g$ satisfying (a) and (b), with $f \notin C^{[\alpha]+1}$.

Proof. (i) Let us show first that if $f$ is real, then (a) and (b) imply $f \in C^{[2 \alpha]}$. Denote by $P$ the (closed) set of flatness of $g$. Then $f$ is flat on $P($ by $(b))$ and smooth on $\boldsymbol{R} \backslash P$ (by Lemma 1). In particular $f$ is continuous. Suppose that $f \in C^{n-1}$ and $p \in P$. Then

$$
(g f)^{(n+2)}(x) \longrightarrow 0 \quad \text { as } \quad x \rightarrow p, \quad x \notin P,
$$

that is, by Leibniz' formula,

$$
\begin{aligned}
g(x) f^{(n+2)}(x)+(n+2) g^{\prime}(x) f^{(n+1)}(x) & +\left(\begin{array}{c}
n+2 \\
2
\end{array}\right) g^{\prime \prime}(x) f^{(n)}(x) \\
& +\sum_{j=o}^{n-1}\left(\begin{array}{c}
n+2 \\
j
\end{array}\right) g^{(n+2-j)}(x) f^{(j)}(g) \longrightarrow 0 .
\end{aligned}
$$

But $g^{(n+2-j)}(x) f^{(j)}(x) \rightarrow 0$ if $j \leqq n-1$, by Lemma 2 . Let

$$
\varphi(x)=f^{(n)}(x) \quad \text { for } \quad x \notin P,
$$

Then

$$
\left(g \varphi^{\prime \prime}\right)(x)+(n+2)\left(g^{\prime} \varphi^{\prime}\right)(x)+\left(\begin{array}{c}
n+2 \\
2
\end{array}\right)\left(g^{\prime \prime} \varphi\right)(x) \longrightarrow 0,
$$

as $p \in P, x \rightarrow p, x \notin P$. Suppose now that

$$
n \leqq 2 \alpha, \quad f \in C^{n-1} \backslash C^{n} .
$$

We shall prove that this is inconsistent with (36).

LEMMA 12. If the function $f:[q, a] \rightarrow \boldsymbol{R}$ is flat at $q$ and $m$-times differentiable for $x>q$, then $\liminf _{x \rightarrow q}\left|f^{(m)}(x)\right|=0$.

Proof. We may suppose that $q=0$. By Lemma 3 and the flatness of $f$ at 0 , we get for $0<s \leqq a / 2$ :

$$
\inf _{0<x<2 s}\left|f^{(m)}(x)\right| \leqq \inf _{s \leqq x \leqq 2 s}\left|f^{(m)}(x)\right| \ll s^{-m} \sup _{s \leqq x \leqq 2 s}|f(x)| \ll s^{-m} \cdot s^{m+1}=s .
$$


This proves the lemma.

Q.E.D.

LEMMA 13. Let $P$ be a closed set in $\boldsymbol{R}$, and let $f: \boldsymbol{R} \rightarrow \boldsymbol{R}$ be a real function, smooth on $\boldsymbol{R} \backslash P$ and flat on $P$. Suppose that $f \in C^{n-1} \backslash C^{n}$ for some natural number n. Then there exist $p \in P$, and a sequence of intervals $I_{k}=\left[a_{k}, b_{k}\right] \subset \boldsymbol{R} \backslash P$ with $a_{k}<b_{k}, a_{k} \rightarrow p, b_{k} \rightarrow p$, and a sequence of numbers $c_{k} \in I_{k}$, such that

$$
\begin{gathered}
\sup _{I_{k}}|f| \gg\left|I_{k}\right|^{n} ; \\
\sup _{I_{k}}\left|f^{(n)}\right| \gg 1 ; \\
\sup _{I_{k}}\left|f^{(n+1)}\right|=\left|f^{(n+1)}\left(c_{k}\right)\right| \geqq \frac{1}{2}\left|I_{k}\right|^{-1} \sup _{I_{k}}\left|f^{(n)}\right| ; \\
f^{(n+2)}\left(c_{k}\right)=0 ; \\
\sup _{I_{k}}\left|f^{(n+2)}\right| \geqq\left|I_{k}\right|^{-1} \sup _{I_{k}}\left|f^{(n+1)}\right| .
\end{gathered}
$$

Proof. By the remark which follows Lemma 7, we may find $p \in P$ and a sequence of numbers $y_{k} \notin P, y_{k} \rightarrow p$, such that (with $\varphi=f^{(n)}$ as above),

$$
\varphi\left(y_{k}\right) \geqq c>0 \text {. }
$$

(Henceforth, we shall replace sequences by subsequences if necessary, without mentioning it each time.) We may suppose $p=0, y_{k}>0, \varphi\left(y_{k}\right) \geqq 2$. There are $u_{k}, 0<u_{k}<y_{k}$, such that $\left[u_{k}, y_{k}\right] \subset \boldsymbol{R} \backslash P$ and

$$
\varphi\left(u_{k}\right)=1, \quad \varphi(x) \geqq 1 \quad \text { in }\left[u_{k}, y_{k}\right] .
$$

This follows from Lemma 12 with $q=\sup \left(P \cap\left[0, y_{k}\right]\right), a=y_{k}, n=m$. We may suppose $y_{k}<u_{k-1}$. Then, there is a sequence of numbers $z_{k}, y_{k}<z_{k} \leqq u_{k-1}$ such that $\left[y_{k}, z_{k}\right] \subset \boldsymbol{R} \backslash P$,

$$
\varphi\left(z_{k}\right)=1, \quad \varphi(x) \geqq 1 \quad \text { in }\left[y_{k}, z_{k}\right] .
$$

This follows from (43) and from $\varphi\left(u_{k-1}\right)=1$ if $\left[y_{k}, u_{k-1}\right] \subset \boldsymbol{R} \backslash P$, and again from Lemma 12 in the opposite case. Now we choose $b_{k}$ such that $u_{k} \leqq b_{k} \leqq z_{k}$ and

$$
\varphi^{\prime}\left(b_{k}\right)=\max _{\left[u_{k}, z_{k}\right]} \varphi(x) \geqq 2, \quad \varphi^{\prime}\left(b_{k}\right)=0 .
$$

Set $J_{k}=\left[u_{k}, b_{k}\right]$. Then $\varphi \geqq 1$ in $J_{k}$ by (43) and (44); by Lemma 3 we then obtain

$$
\sup _{J_{k}}|f| \geqslant\left|J_{k}\right|^{n} \text {. }
$$

If $\left|\varphi^{\prime}\right|$ has a maximum on $J_{k}$ at a point $c_{k}$ where $\varphi^{\prime \prime}$ vanishes, we set $a_{k}=u_{k}$, $I_{k}=J_{k}$; then (40) follows from

$$
\sup _{J_{k}}|\varphi| \geqq 2 \inf _{J_{k}}|\varphi|,
$$


and (42) in a similar way. In the opposite case, $\left|\varphi^{\prime}\right|$ has its maximum in $J_{k}$ at $u_{k}$, and $\varphi^{\prime}\left(u_{k}\right) \geqq\left|J_{k}\right|^{-1}$, for (43) implies $\varphi^{\prime}\left(u_{k}\right) \geqq 0$. Using Lemma 12 once more we find a point $a_{k}, 0<a_{k}<u_{k},\left[a_{k}, u_{k}\right] \subset \boldsymbol{R} \backslash P$, such that

$$
\varphi^{\prime \prime}\left(a_{k}\right)=0, \quad \varphi^{\prime}\left(a_{k}\right) \geqq \varphi^{\prime}(x) \geqq \varphi^{\prime}\left(u_{k}\right) \geqq\left|J_{k}\right|^{-1} \text { for } a_{k} \leqq x \leqq u_{k} .
$$

Set $I_{k}=\left[a_{k}, b_{k}\right]$ and $c_{k}=a_{k}$. The estimates (39)-(42) follow as above. In order to prove (38), we distinguish two cases $u_{k}-a_{k} \leqq b_{k}-u_{k}$ and $u_{k}-a_{k} \geqq b_{k}-u_{k}$. In the first case we have $\left|I_{k}\right| \leqq 2\left|J_{k}\right|$, and (38) is a consequence of (46). In the second case, we obtain (using Lemma 3 with (48)):

$$
\sup _{\left[a_{k}, u_{k}\right]}|f| \gg\left(u_{k}-a_{k}\right)^{n+1} \inf _{\left[a_{k}, u_{k}\right]} \varphi^{\prime} \geqq\left|J_{k}\right|^{-1}\left(u_{k}-a_{k}\right)^{n+1} \geqq\left|I_{k}\right|^{-1}\left(\frac{1}{2}\left|I_{k}\right|\right)^{n+1},
$$

thus proving (38).

Q.E.D.

Let us go back to the proof of Theorem 7. We suppose that (37) is true. By (38) and the hypothesis (b), we obtain

$$
\sup _{I_{k}}|g| \gg\left|I_{k}\right|^{n / \alpha} \text {. }
$$

We apply Lemma 5 with $\imath=n / \alpha \leqq 2$, the $n$ of the lemma being 0 . Thus there is a number $m<n / \alpha$ such that

$$
\inf _{I_{k}}\left|g^{(m)}\right| \gg\left|I_{k}\right|^{n / \alpha-m}
$$

and

$$
\sup _{I_{k}}\left|g^{(l)}\right|=o\left(\left|I_{k}\right|^{n / \alpha-l}\right) \quad \text { for } \quad l>m \text {. }
$$

Since $n / \alpha \leqq 2$, one has $m=0$ or $m=1$.

In the first case $(m=0)$ we obtain from (39), (40), (42), (49) and (50) (setting $\sup =\sup _{I_{k}}, \inf =\inf _{I_{k}}$ for simplicity):

$$
\begin{aligned}
& \sup \left|g \varphi^{\prime \prime}+(n+2) g^{\prime} \varphi^{\prime}+\left(\begin{array}{c}
n+2 \\
2
\end{array}\right) g^{\prime \prime} \varphi\right| \\
& \geqq \sup \left|\varphi^{\prime \prime}\right| \inf |g|-(n+2)^{2}\left(\sup \left|\varphi^{\prime}\right| \sup \left|g^{\prime}\right|+\sup |\varphi| \sup \left|g^{\prime \prime}\right|\right) \\
& \geqq \sup \left|\varphi^{\prime \prime}\right|\left(\inf |g|-\left|I_{k}\right| o\left(\left|I_{k}\right|^{n / \alpha-1}\right)-\left|I_{k}\right|^{2} o\left(\left|I_{k}\right|^{n / \alpha-2}\right)\right) \\
&\left.\gg\left|I_{k}\right|^{-2}\left|I_{k} !^{n / \alpha}=\right| I_{k}\right|^{n / \alpha-2} \gg 1,
\end{aligned}
$$

which is inconsistent with (36). If $m=1$, we have

$$
\begin{array}{r}
\left|\left(g \varphi^{\prime \prime}+(n+2) g^{\prime} \varphi^{\prime}+\left(\begin{array}{c}
n+2 \\
2
\end{array}\right) g^{\prime \prime} \varphi\right)\left(c_{k}\right)\right| \\
=\left|\left((n+2) g^{\prime} \varphi^{\prime}+\left(\begin{array}{c}
n+2 \\
2
\end{array}\right) g^{\prime \prime} \varphi\right)\left(c_{k}\right)\right|
\end{array}
$$




$$
\begin{aligned}
& \geqq(n+2)\left|\varphi^{\prime}\left(c_{k}\right)\right|\left(\inf \left|g^{\prime}\right|-(n+1)\left|I_{k}\right| \sup \left|g^{\prime \prime}\right|\right) \\
& \gg\left|I_{k}\right|^{-1}\left(\left|I_{k}\right|^{n / \alpha-1}-\left|I_{k}\right| o\left(\left|I_{k}\right|^{n / \alpha-2}\right)\right) \\
& \gg\left|I_{k}\right|^{n / \alpha-2} \gg 1,
\end{aligned}
$$

which is again inconsistent with (36). This time, (41) was used instead of (42). This completes the proof of $f \in C^{[2 \alpha]}$.

(ii) If $f$ is complex, then by hypothesis $g \in C^{\infty}$, therefore $|g|^{2}=\bar{g} g \in C^{\infty}$ and $f|g|^{2}=(f g) \bar{g} \in C^{\infty}$, thus $\Re f|g|^{2} \in C^{\infty}$. Condition (b) becomes $|\Re f| \leqq\left(|g|^{2}\right)^{\alpha / 2}$, it follows (by the real case) that $\Re f \in C^{[2 \alpha / 2]}=C^{[\alpha]}$. Similarly, $\Im f \in C^{[\alpha]}$, and finally $f \in C^{[\alpha]}$ ( $\mathfrak{Q}$ stands for "imaginary part of").

It is possible to give a direct proof, similar to (i). We can obtain an analogous of Lemma 13, but without (41) which we needed to treat the case $m=1$; to exclude this case we have to suppose $n / \alpha \leqq 1$, that is to say $n \leqq[\alpha]$.

(iii) We choose a function $H \in C^{\infty}(\boldsymbol{R}, \boldsymbol{R})$ having the properties

$$
\begin{gathered}
0 \leqq H \leqq 1, \quad H(y)=0 \quad \text { for }|y| \geqq 1 ; \\
H-1 \text { is flat at } y=0 .
\end{gathered}
$$

We then choose a non-constant (real or complex) polynomial $p$ of degree $N$ with the property

$$
|p(y)| \geqq 1 \text { for } y \text { real. }
$$

$1 / p$ is not a polynomial because $p$ is not constant. Therefore we may suppose that

$$
\left(\frac{d}{d y}\right)^{[N \alpha]+1}\left(\frac{1}{p}\right)(0) \neq 0 .
$$

Finally we choose two sequences $c_{1}>c_{2}>c_{3}>\cdots>0, c_{k} \rightarrow 0$, and $D_{1}, D_{2}, D_{3}, \cdots$ with $0<D_{k} \leqq 1 / 2 c_{k}, c_{k+1}+D_{k+1}<c_{k}-D_{k}$, and set $d_{k}=\exp \left(-1 / D_{k}\right), I_{k}=\left[c_{k}-D_{k}, c_{k}+D_{k}\right]$. The $I_{k}$ are disjoint and accumulate towards 0 . With

$$
\beta=[N \alpha]+1, \quad \gamma=\beta / \alpha, \quad \omega=[\alpha]+1,
$$

we define $f, g: \boldsymbol{R} \rightarrow C$ by

$$
\begin{aligned}
& g(x)=d_{k}^{\gamma} p\left(\left(x-c_{k}\right) / d_{k}\right) H\left(\left(x-c_{k}\right) / D_{k}\right) \text { in } I_{k} ; \\
& f(x)=d_{k}^{\beta} \frac{H^{\omega}\left(\left(x-c_{k}\right) / D_{k}\right)}{p\left(\left(x-c_{k}\right) / d_{k}\right)} \text { in } I_{k} .
\end{aligned}
$$

$f(x)=g(x)=0$ if $x$ is not in the union of the $I_{k}$, in particular $f(0)=g(0)=0$. From $|p| \geqq 1,|H| \leqq 1$ and $\alpha<\omega$ we obtain

$$
|g(x)|^{\alpha} \geqq d_{k}^{\alpha \gamma} H^{\alpha}\left(\left(x-c_{k}\right) / D_{k}\right) \geqq d_{k}^{\beta} H^{\omega}\left(\left(x-c_{k}\right) / D_{k}\right) \geqq|f(x)|,
$$

for $x \in I_{k}$, and hence for all $x \in \boldsymbol{R}$; thus condition (b) of the theorem holds. Because $\gamma>N$ and $d_{k} \ll D_{k}^{S}$ for arbitrary $S>0$, we have 


$$
\begin{aligned}
g^{(n)}(x) & =\sum_{0}^{n}\left(\begin{array}{l}
n \\
m
\end{array}\right) d_{k}^{\gamma-m} p^{(m)}\left(\left(x-c_{k}\right) / d_{k}\right) D_{k}^{m-n} H^{(n-m)}\left(\left(x-c_{k}\right) / D_{k}\right) \\
& \ll \sum_{m=0}^{\min (n, N)} d_{k}^{\gamma-m}\left(D_{k} / d_{k}\right)^{N-m} D_{k}^{m-n} \\
& \ll d_{k}^{\gamma-N} D_{k}^{N-n} \ll D_{k}^{M},
\end{aligned}
$$

if $x \in I_{k}$, for $n=0,1,2, \cdots, M>0$, with constants depending on $n$ and $M$. Since $x \geqq D_{k}$ for $x \in I_{k}$, we can easily deduce that $g \in C^{\infty}$ and that $g$ is flat at $x=0$ ( $f$ is therefore also flat at 0 ). Similarly, one shows that $f g \in C^{\infty}$. On the other hand we get

$$
f^{(\beta)}\left(c_{k}\right)=\left(p^{-1}\right)^{(\beta)}(0) \neq 0,
$$

by (52) and (54) and therefore $f^{(\beta)}\left(c_{k}\right) \gg 1$. Since $f$ is flat at 0 , we deduce that $f \notin C^{\beta}=C^{[N \alpha]+1}$. As a particular case let us choose $N=2, p(y)=1+(y-s)^{2}$, where $s \in \boldsymbol{R}$ is such as to satisfy (54). Then $f$ and $g$ are also real and $f \notin$ $C^{[2 \alpha]+1}$. If we choose $N=1, p(y)=1+i(y-s)$, then $f$ and $g$ are complex and $f \notin C^{[\alpha]+1}$. This completes the proof.

Q.E.D.

\section{REFERENCES}

[AM] I. Anemiya and K. Masuda, On Joris' theorem on differentiability of functions, Kodai Mathematical Journal, 12 (1989), pp. 92-97.

[DKP] J. Duncan, S. G. Krantz and H. R. Parks, Non-linear Conditions for Differentrability of Functions, Journal d'Analyse Math., 45 (1985), pp. 46-68.

[F] G.M. Fichtenholz, Differential- und Integralrechnung Bd. 1, VEB Deutscher Verlag der Wissenschaften Berlin, 1981.

[J ] H. JoRIs, Une $C^{\infty}$-application non-1mmersive qui possède la propriété universelle des immersions, Archiv der Mathematik, 39 (1982), pp. 269-277.

[JP1] H. Joris and E. Preissmann, Pseudo-immersions, Annales de l'Institut Fourier 37 (1987), pp. 195-221.

[JP2] H. JoRis and E. Preissmann, Germes pseudo-immersifs de $\left(\boldsymbol{R}^{n}, 0\right)$ dans $\left(\boldsymbol{R}^{n}, 0\right)$, C.R. Acad. Sci. Parıs, t. 305, Série I (1987), pp. 261-263.

[L] S. Lojasiewicz, Sur le problème de la division, Studia Mathematica 18 (1959), pp. $87-136$.

[M] B. Malgrange, Ideals of differentiable functions, Oxford University Press (Tata Institute Publications), 1966.

[N] R. Narasimhan, Complex Analysis in One Variable, Birkhäuser, Boston, 1985.

[S] I. R. Shafarevich, Basic Algebraic Geometry, Springer, Berlin, 1974.

[W] A. WeIL, Basic Number Theory, Springer, Berlin, 1967.

[ZS] O. Zariski and P. Samuel, Commutative Algebra Vol. 1, Springer, Berlin, 1979, (Van Nostrand, Princeton, 1958.)

Adress of Authors: Institut de Mathématiques,

Faculté des Sciences, Université de Lausanne,

Collège Propédeutique, Ch-1015 Lausanne-Dorigny, Suisse. 